\title{
Statistical support for the hypothesis of developmental constraint in marsupial skull evolution
}

\author{
C Verity Bennett $^{1^{*}}$ and Anjali Goswami ${ }^{1,2}$
}

\begin{abstract}
Background: In contrast to placental neonates, in which all cranial bones are ossified, marsupial young have only the bones of the oral region and the exoccipital ossified at birth, in order to facilitate suckling at an early stage of development. In this study, we investigated whether this heterochronic shift in the timing of cranial ossification constrains cranial disparity in marsupials relative to placentals.
\end{abstract}

Methods: We collected three-dimensional (3D) landmark data about the crania of a wide range of extant placentals and marsupials, and from six fossil metatherians (the clade including extant marsupials and their stem relatives), using a laser scanner and a 3D digitizer. Principal components analysis and delta variance tests were used to investigate the distribution and disparity of cranial morphology between different landmark sets (optimizing either number of landmarks or number of taxa) of the whole skull and of individual developmental or functional regions (neurocranium, viscerocranium, oral region) for extant placentals and marsupials. Marsupial and placental data was also compared based on shared ecological aspects including diet, habitat, and time of peak activity.

Results: We found that the extant marsupial taxa investigated here occupy a much smaller area of morphospace than the placental taxa, with a significantly $(P<0.01)$ smaller overall variance. Inclusion of fossil taxa did not significantly increase the variance of metatherian cranial shape. Fossil forms generally plotted close to or within the realm of their extant marsupial relatives. When the disparities of cranial regions were investigated separately, significant differences between placentals and marsupials were seen for the viscerocranial and oral regions, but not for the neurocranial region.

Conclusion: These results support the hypothesis of developmental constraint limiting the evolution of the marsupial skull, and further suggest that the marsupial viscerocranium as a whole, rather than just the earlyossifying oral region, is developmentally constrained.

Keywords: Marsupial, Placental, Cranium, Developmental constraint, Geometric morphometrics

\section{Background}

Fossil and molecular estimates generally agree that the lineages leading to marsupial and placental mammals diverged over 160 million years ago (Ma), in the Late Jurassic period $[1,2]$. Despite this shared time of origin as sister clades, recent marsupials and placentals differ markedly in taxonomic diversity and geographical range. Whereas placentals number over 5,000 species and are globally distributed,

\footnotetext{
* Correspondence: ucbtcvb@ucl.ac.uk

'Department of Genetics, Evolution and Environment, University College London, London, UK

Full list of author information is available at the end of the article
}

extant marsupials are far less speciose, with 331 species, and occupy only Australasia, South America, and Central America, with one species in North America [3], yet the marsupial (and broader metatherian) fossil record demonstrates that this now depauperate and geographically restricted clade previously had a global distribution [4-7]. The differences in the evolutionary histories of these two clades, and how these differences have contributed to their disparate modern diversities, has been a topic of research and debate for decades, but there is as yet little consensus on the relative importance of intrinsic factors such as development, and extrinsic drivers such as competition or

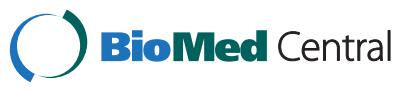


geography [8-10]. In this study, we investigated the evidence for the role of developmental constraints on marsupial evolution.

The most obvious difference between marsupial and placental mammals is, as their clade names suggest, their developmental mode. Marsupials are born in a highly altricial state, and must immediately travel to their mother's teat, which is often located in the pouch [11]. Once they attach to a nipple, marsupial young undergo a much longer period of development. The nature of the obligatory, independent journey to the pouch varies across marsupial clades. For didelphimorphians (opossums) and some diprotodontians (including possums and kangaroos), which have forwardfacing pouches, an upwards, forelimb-powered crawl is required. In peramelimorphians (bandicoots) and some diprotodontians (such as wombats), which have backwardsfacing pouches, and in dasyuromorphians (marsupial carnivores and mice), which have open pouches, the journey to the pouch is a downwards, sinusoidal slither, aided by the positioning of the mother (Wilson and Reeder [3] and references therein). These mechanical demands do not end with arrival in the pouch, as once the neonate is attached to the teat, it must satisfy the mechanical demands of suckling to survive. To fulfill this function, marsupial skulls at birth are necessarily ossified in the oral region (including the anterior portion of the mandible, premaxillae, maxillae, palatines, and pterygoids) for feeding, and the exoccipital region for movement of the head relative to the spine, whereas the remaining cranial bones ossify after birth [12]. By contrast, even the most altricial neonates of their placental sister group are born at a much later stage of development, with all or nearly all cranial bones at least partially ossified prior to the commencement of suckling.

It has long been hypothesized and debated that early functional demands have constrained the evolution of novel morphologies in marsupial phenotypes $[9,13,14]$ with particular regard to the lack of fully volant or aquatic marsupial species. In a study quantifying ontogenetic changes in the shoulder girdle and comparing adult diversity in the scapula and pelvis, Sears [15] found evidence for constraint in marsupial shoulder-girdle morphology produced by this early functional requirement. There is also evidence that the early crawl constrains forelimb morphology in marsupials [16,17].

Whether or not the morphology of the marsupial skull is also constrained by these early functional demands requires the comparison of adult morphology, the end product of development. A few previous studies have quantitatively compared disparity in adult cranial morphologiy across placentals and marsupials, but all of these have focused almost exclusively on carnivorous taxa [18-21]. Goswami et al. [21] found no evidence for cranial constraint when comparing the adult morphological variance of extant and extinct metatherian and eutherian hypercarnivores, although the early-ossifying oral apparatus was not assessed separately. By contrast, Prevosti et al. [22] found that disparity in mandible morphology is more constrained in extant carnivorous marsupials than in the Carnivora; however, the exclusion of extinct forms from that analysis left the most specialized marsupial carnivores unsampled. Marsupial ecology extends far beyond carnivory. For example, members of Diprotodontia, the most taxonomically diverse marsupial order today, are mostly folivores (including browsers and grazers), although there are also many frugivores, insectivores, and omnivores, and, in the recent past, carnivores within this clade. Whether the skull or mandible shows evidence for developmental constraint in marsupials representing ecological groups other than carnivores has yet to be tested.

In this study, we quantitatively tested the hypothesis of developmental constraint in the marsupial cranium across marsupial phylogeny and across diverse ecologies (diet, habitat, and time of activity) using geometric morphometrics. It is important to consider all of these aspects and to sample the full range of marsupial ecology in order to make meaningful comparisons between marsupial and placental diversity. Specifically, we tested whether marsupials show significantly less cranial disparity than placentals across the entire skull and within relevant developmental and functional sub-regions (viscerocranium, neurocranium, oral apparatus). The independent comparison of cranial sub-regions allows assessment of whether any observed differences in disparity between marsupials and placentals are driven specifically by the early-ossifying regions of the skull (that is, the oral apparatus). We further test whether the addition of well-preserved fossil metatherians to the dataset would significantly increase the disparity measured from extant marsupials alone.

\section{Results}

\section{Principal components analysis}

The following results describe the first four principal components (PCs) of each analysis, as subsequent PCs did not explain a sufficiently large percentage of the variation to warrant meaningful discussion (see Additional file 1: Table S1).

In the extant-only 'maximum landmarks' dataset (Figure 1) PC1 (35\% of the variance) separated the longsnouted, narrow skulls of peramelemorphians from the flat-faced, wider and taller skulls of primates. PC2 in this dataset (12\% of the variance) separated the longer, dorsoventrally shorter skulls of artiodactyls from the taller, anterioposteriorly shorter skulls of diprotodontians. Using these first two PCs, many diprotodontians and a few didelphimorphians fell outside of placental space, but all dasyuromorphians fell within the range of placental morphospace. PC3 and PC4, each accounted for $8 \%$ of 


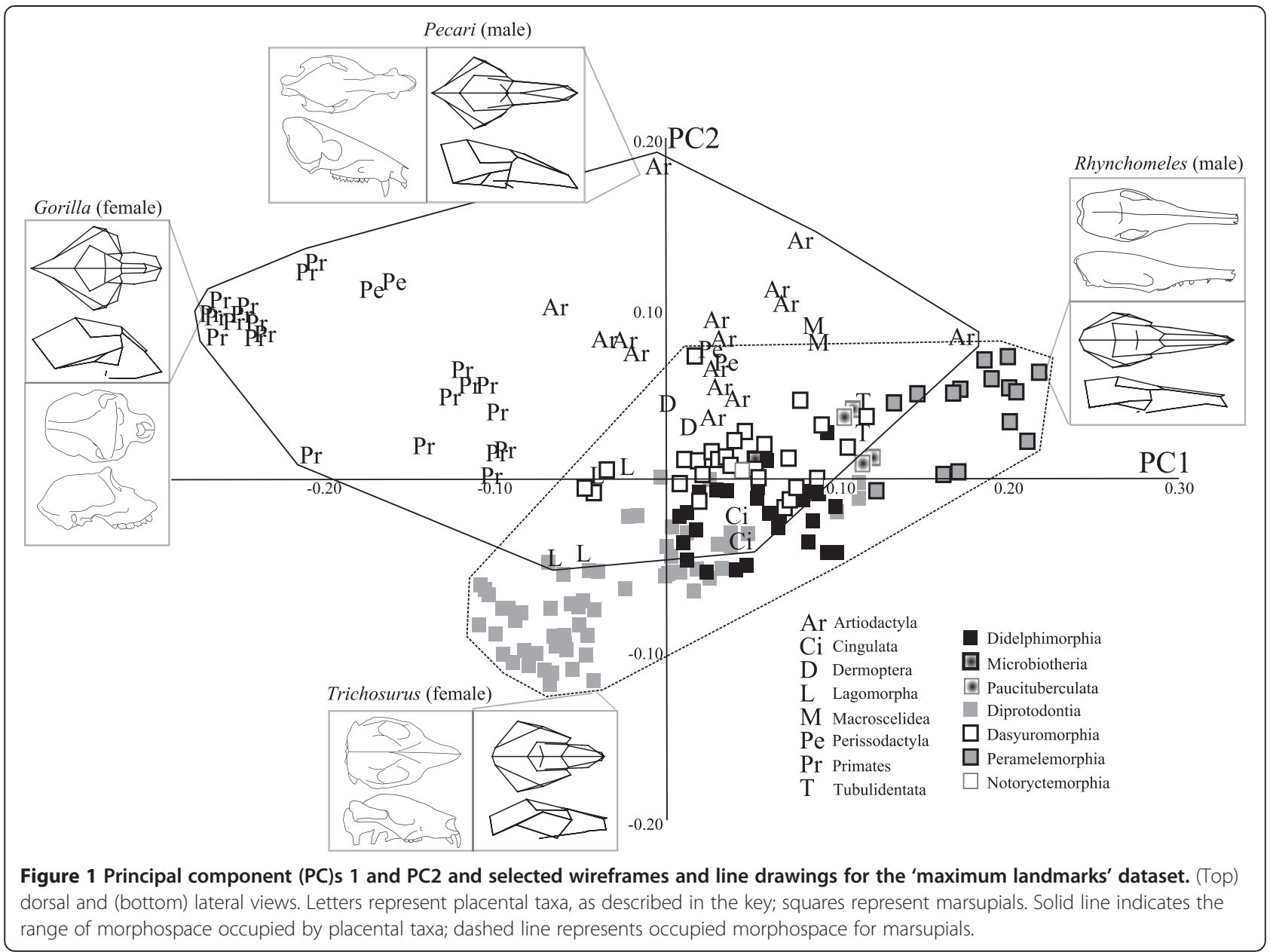

the total variance (Figure 2), showed far less phylogenetic clustering and greater overlap between orders. On these axes, marsupial morphospace was entirely within placental space.

In the 'maximum taxa' dataset (Figure 3), PC1 accounted for $39 \%$ of the variance, and showed the same separation between long-snouted and flat-faced skulls as in the 'maximum landmarks' dataset. PC2 (14\% of the variance) separated the long-snouted, narrower skulls of the pangolin from the wider, taller skulls of diprotodontians in the "maximum taxa dataset'. PC3 and PC4 (10\% and 7\% of the total variance, respectively; Figure 4) also showed more overlap between marsupial and placental morphospace than PC1 and $\mathrm{PC} 2$, and only a few diprotodontians fell outside of placental space.

Taxa largely clustered by phylogenetic relationship in the morphospace described by these first two PCs for both extant-only datasets. Shape changes on axes 3 and 4. were far subtler than for PC1 and PC2. There was extensive overlap between the marsupial and placental morphospaces in both datasets, and placentals occupied a larger area of morphospace in all PC analysis plots.
The placental orders Afrosoricida, Tubulidentata, Pholidota, Lagomorpha, Hyracoidea, Cingulata, Dermoptera, Scandentia, Rodentia, Erinaceomorpha, and Macroscelidea all fell within or very close to the region of the morphospace occupied by marsupials. Some artiodactyls and perissodactyls also fell near marsupials in the major axes of the morphospace. Although there was some overlap, Primates and Carnivora fell furthest away from the marsupials in both analyses.

When the 'maximum taxa' dataset was subdivided into ecological groupings, marsupials again inhabited a relatively smaller region of morphospace on $\mathrm{PC} 1$ to $\mathrm{PC4}$, and overlapped entirely with placentals (see Additional file 2: Figure S2; see Additional file 3: Figure S3; see Additional file 4: Figure S4) for nearly all the ecological groups. The sole exception to this pattern was found in the analysis of fossorial taxa, in which placentals and marsupials occupied roughly equal areas of morphospace.

\section{Delta variance tests}

Both the 'maximum taxa' and 'maximum landmarks' datasets showed significantly greater $(P<0.01)$ morphological disparity 


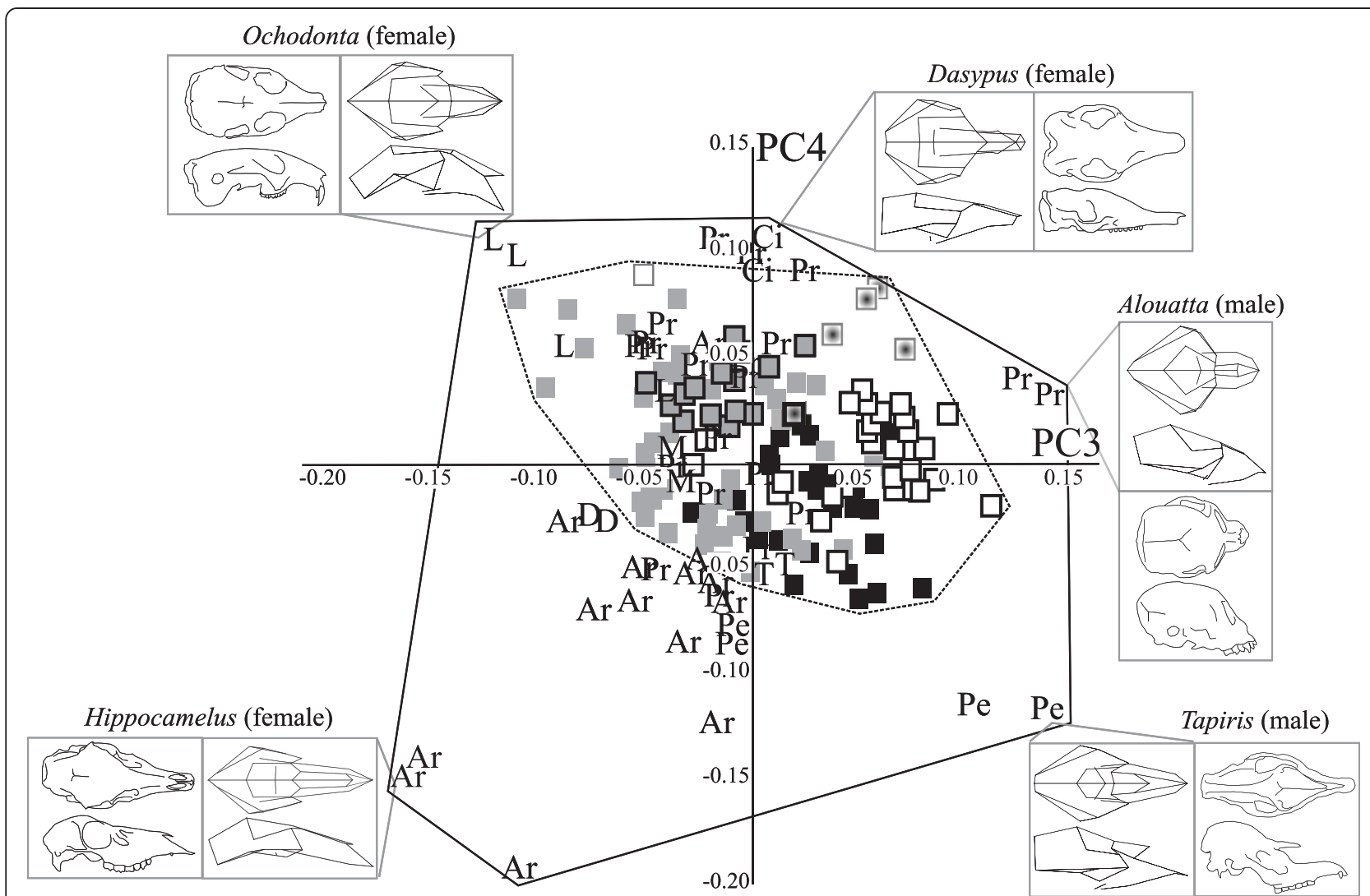

Figure 2 Principal components (PC)3 and 4 and selected wireframes and line drawings for the 'maximum landmarks' dataset. (Top) dorsal and (bottom) lateral views. Symbols as in Figure 2.

(higher variance) in placentals than in marsupials when the full skull was considered (Table 1). In the comparison of developmentally and functionally significant cranial sub-regions, the viscerocranium including the oral region, the viscerocranium excluding the oral region, and the oral region alone all showed significantly $(P<0.01)$ greater morphological disparity in placentals than marsupials, and these differences remained after Bonferroni correction. However, there was no significant difference between marsupials and placentals in disparity of the neurocranial region.

Similar results were obtained when taxa were divided into ecological groups, with the exception of marginally significant differences in neurocranial disparity between arboreal $(P=0.03)$ and folivorous $(P=0.048)$ marsupials and placentals; however, these exceptions were not supported after Bonferroni correction. There were marginally significant differences between placentals and marsupials in the disparity of the entire skull for folivorous $(P=$ $0.027)$ and carnivorous $(P=0.034)$ forms, but again, not after Bonferroni correction, whereas all other ecological groups showed significantly different $(P<0.01)$ disparity between marsupials and placentals. All three groups of viscerocranial landmarks showed significantly higher disparity in placentals than in marsupials when ecological groups were compared separately, with the exception of fossorial forms. Fossorial marsupials and placentals showed no significant difference in viscerocranial disparity, except for a marginally significant difference (before Bonferroni correction) in the oral region $(P=0.033)$. After Bonferroni correction, no set of viscerocranial landmarks showed significant differences between fossorial marsupials and placentals.

\section{Fossil taxa}

When fossil marsupials are added into the analysis, five of the six fossil taxa were found to fall outside the region of morphospace of PC1 (37\% variance) and PC2 (18\% variance) occupied by Recent marsupials (Figure 5). Galadi, the Oligo-Miocene bandicoot, falls with other peramelemorphians, whereas Sthenurus falls close to other diprotodontians. The remaining fossil diprotodontians, Thylacoleo and Zygomaturus, plotted more distantly to other diprotodontians, and the sparassodont Arctodictis plots very closely to Zygomaturus, and much farther from the only other sparassodont included in this study, the sabre-toothed marsupial Thylacosmilus atrox. Inclusion of these fossil taxa with recent forms did not significantly 


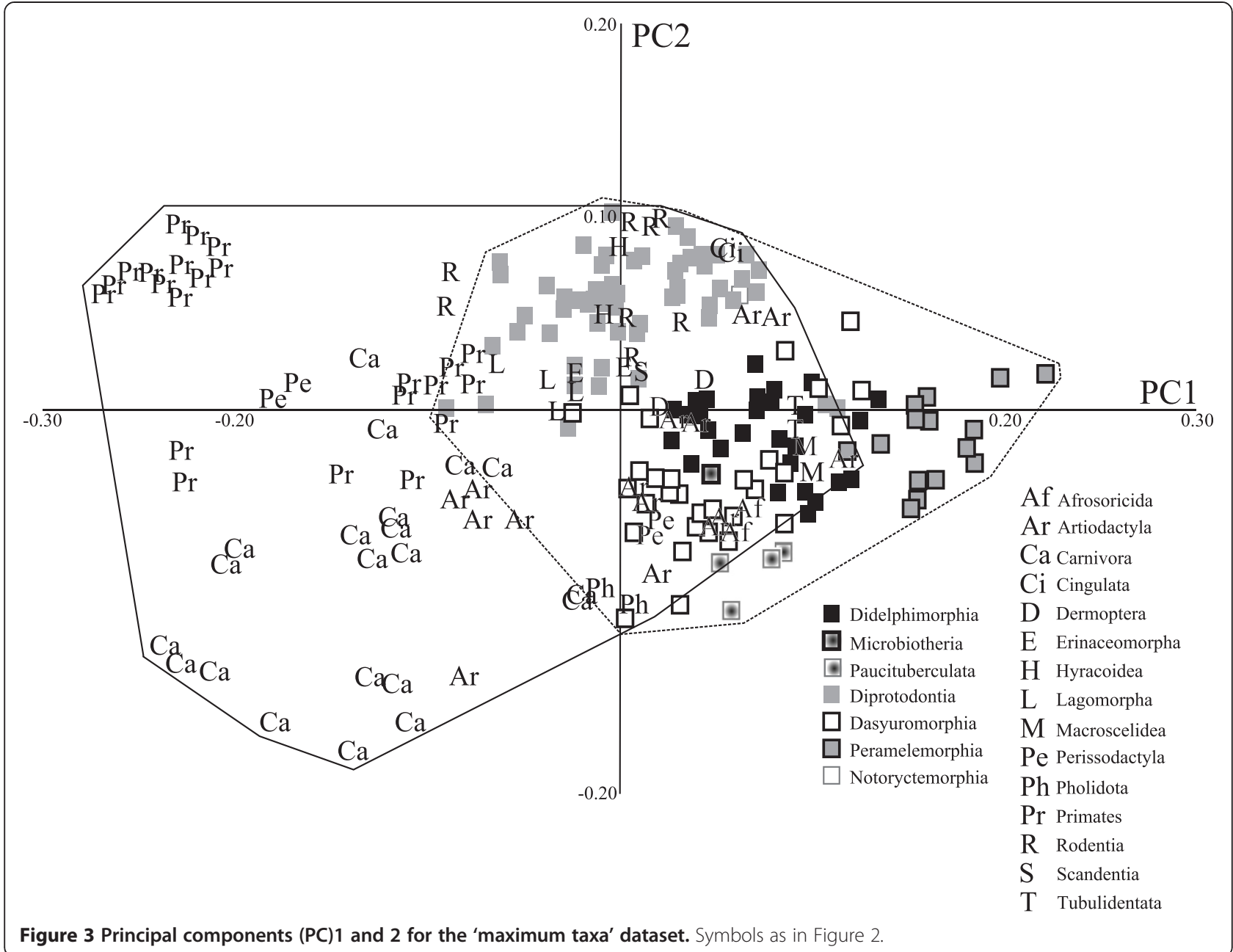

increase the morphological variance of the metatherian dataset (Table 2) or change the results of the delta variance permutation tests comparing marsupial (and nonmarsupial metatherian) and placental disparity.

\section{Discussion}

Despite the exclusion of several placental taxa with unusual cranial morphologies (notably whales, bats, and elephants), and the limited fossil taxa included, we found in the current study that marsupial crania are, on the whole, significantly less disparate compared with placentals. Although inclusion of the enigmatic Tarsipes could potentially increase the variance of the extant marsupials in future studies, we are confident that this one taxon would not alter the substantial difference in variance between marsupials and placentals reported here. Thus, the results of this study support the hypothesis that marsupial crania are developmentally constrained, and that this constraint is likely to have limited the morphological evolution of marsupials relative to their placental sister groups. In particular, the observation that the viscerocranial region, which includes the early-ossifying bones of the oral region, is significantly less disparate in marsupials than in placentals, whereas the late-ossifying neurocranial region has similar disparity in both clades, is consistent with the hypothesis that the differential evolutionary success of these two groups was shaped by developmental strategy rather than by extrinsic factors.

\section{Developmental timing, integration, and lability}

Although relative cranial ossification sequence is largely conserved across mammals $[23,24]$, there is a delay in raw timing between the development of bones in the oral region and those in the neurocranium of marsupials compared with placentals [25-27]. Anterior elements of the skull also tend to show less heterochronic variation and basicranial elements show the most [23]. As we tested here, these differences in raw timing and rank variability in ossification sequences correlate with differential cranial disparity for the viscerocranial and neurocranial regions, with the former showing significantly less disparity in marsupials than in placentals. These regional differences 


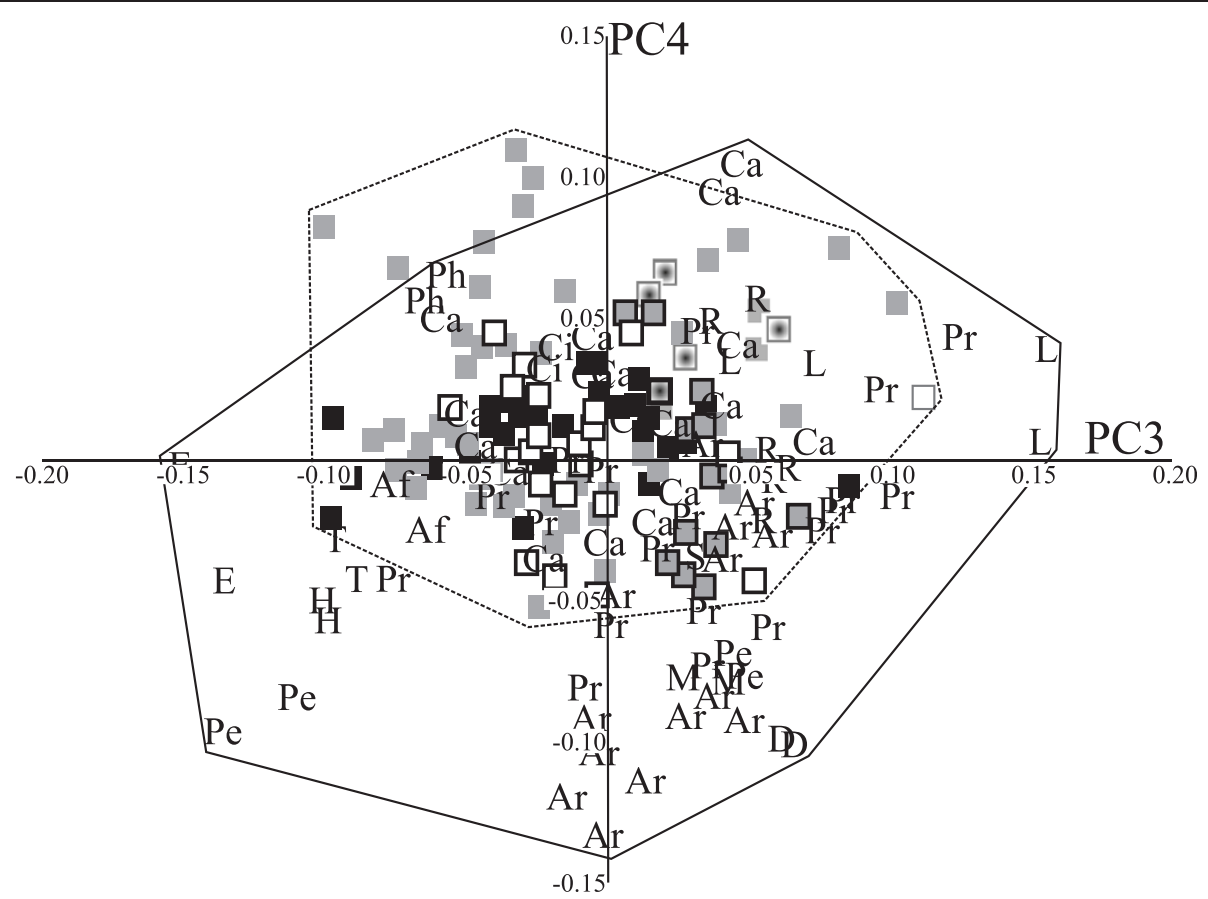

Figure 4 Principal components (PC)3 and 4 for the 'maximum taxa' dataset. Symbols as in Figure 2.

in amount of heterochronic variation may possibly relate to the different evolutionary lability of these regions [23], although this hypothesis has yet to be tested with quantitative data on ontogenetic or morphological variation.

Post-weaning ontogeny of marsupial cranial morphology has been studied in several omnivorous and carnivorous species [28-32]. These studies have shown the existence of some common developmental patterns across marsupials, including a faster-growing viscerocranium than neurocranium in early post-weaning development, negative allometry across the entire braincase and in the height of the occipital plate, and positive allometry in the height of the dentary [31]. That these major aspects of postweaning growth differentiate skull regions is suggestive of the modular nature of cranial development [33-35]. Moreover, that a common growth pattern was found across the full viscerocranium, rather than only in the early-ossifying oral bones of this region, suggests that this region is developmentally integrated, and provides a possible mechanism by which the functional constraints imposed on the oral bones translate to the lower disparity across the entire viscerocranium seen in the current study.

A recent study [35] found that cranial variance in a marsupial (Monodelphis) remained constant through ontogeny, whereas in a placental (Cryptoprocta), variance decreased markedly from the early to the later stages. Moreover, Monodelphis also showed a decrease in integration of the oral region through ontogeny. This led to a tentative hypothesis by the authors that the combination of high integration of the oral region early in ontogeny, alongside functional demands on those early-developing oral bones, may result in low and constant variance of that region through marsupial ontogeny, in contrast to placentals. Although further data are needed to test that hypothesis, a possible extension suggested by the data from the current study could be that, if the marsupial skull is indeed constrained in the oral region during early development, and if the viscerocranial elements of the skull are strongly integrated, as some studies have suggested [36-38], then the remainder of the viscerocranium (for example, those elements that do not ossify early in development) would also be likely to be constrained as a result of integration, rather than by direct developmental or functional constraint.

Not only is the developmental strategy of marsupials very different to that of placentals, but the nature and timing of development also varies between marsupial groups. Peremelemorphs in particular represent the most unusual condition among extant marsupials in having evolved a chorioallantoic placenta, convergent with that of placentals, and also showing the fastest developmental rate of all marsupials [36]. Peramelemorphians also lack a pronounced crawl, as noted above, and this divergent strategy is reflected in their scapular ontogeny, which has been shown to differ significantly from that of other marsupials [15]. Interestingly, the PC analyses presented here show that peramelemorphians fall further outside of placental cranial morphospace than do other marsupial clades. For this reason, we hypothesize that the unusual development of peramelemorphians (which have a much shorter period during which the oral region develops and 
Table 1 Delta variance test results for extant taxa datasets for extant marsupials versus placentals

\begin{tabular}{|c|c|c|c|c|c|}
\hline Dataset & Skull region & Marsupial variance & Placental variance & Delta variance & $P$-value ${ }^{\mathrm{a}}$ \\
\hline \multirow[t]{5}{*}{ Maximum landmarks } & Whole skull & 0.0258 & 0.0681 & 0.0423 & $<<0.001$ \\
\hline & Neurocranium & 0.0344 & 0.0422 & 0.0078 & 0.329 \\
\hline & Viscerocranium & 0.0308 & 0.0873 & 0.0565 & $<<0.001$ \\
\hline & Non-oral viscerocranium & 0.0170 & 0.0446 & 0.0275 & $<<0.001$ \\
\hline & Oral region & 0.0174 & 0.0572 & 0.0398 & $<<0.001$ \\
\hline \multirow[t]{4}{*}{ Maximum taxa } & Whole skull & 0.0178 & 0.0521 & 0.0344 & $<<0.001$ \\
\hline & Neurocranium & 0.0342 & 0.0504 & 0.0162 & 0.150 \\
\hline & Viscerocranium & 0.0180 & 0.0697 & 0.0516 & $<<0.001$ \\
\hline & Oral region & 0.0044 & 0.0251 & 0.0207 & $<<0.001$ \\
\hline \multirow[t]{4}{*}{ Folivores } & Whole skull & 0.0004 & 0.0015 & 0.0011 & $0.027^{b}$ \\
\hline & Neurocranium & 0.0204 & 0.0409 & 0.0205 & $0.048^{b}$ \\
\hline & Viscerocranium & 0.0122 & 0.0649 & 0.0528 & $<<0.001$ \\
\hline & Oral region & 0.0019 & 0.0198 & 0.0179 & $<<0.001$ \\
\hline \multirow[t]{4}{*}{ Frugivores } & Whole skull & 0.0094 & 0.0558 & 0.0464 & 0.001 \\
\hline & Neurocranium & 0.0235 & 0.0433 & 0.0198 & 0.269 \\
\hline & Viscerocranium & 0.0110 & 0.0851 & 0.0740 & 0.006 \\
\hline & Oral region & 0.0019 & 0.0130 & 0.0112 & 0.006 \\
\hline \multirow[t]{4}{*}{ Omnivores } & Whole skull & 0.0130 & 0.0439 & 0.0309 & $<<0.001$ \\
\hline & Neurocranium & 0.0314 & 0.0310 & -0.0004 & 0.966 \\
\hline & Viscerocranium & 0.0092 & 0.0580 & 0.0488 & $<<0.001$ \\
\hline & Oral region & 0.0037 & 0.0347 & 0.0310 & $<<0.001$ \\
\hline \multirow[t]{4}{*}{ Carnivores/insectivores } & Whole skull & 0.0186 & 0.0535 & 0.0348 & $0.034^{b}$ \\
\hline & Neurocranium & 0.0417 & 0.0809 & 0.0392 & 0.280 \\
\hline & Viscerocranium & 0.0165 & 0.0719 & 0.0554 & 0.001 \\
\hline & Oral region & 0.0031 & 0.0225 & 0.0193 & $<<0.001$ \\
\hline \multirow[t]{4}{*}{ Nocturnal/crepuscular } & Whole skull & 0.0181 & 0.0360 & 0.0179 & $<<0.001$ \\
\hline & Neurocranium & 0.0386 & 0.0296 & -0.0090 & 0.413 \\
\hline & Viscerocranium & 0.0186 & 0.0438 & 0.0251 & $<<0.001$ \\
\hline & Oral region & 0.0044 & 0.0198 & 0.0154 & $<<0.001$ \\
\hline \multirow[t]{4}{*}{ Arboreal } & Whole skull & 0.0107 & 0.0347 & 0.0240 & $<<0.001$ \\
\hline & Neurocranium & 0.0156 & 0.0280 & 0.0123 & $0.031^{b}$ \\
\hline & Viscerocranium & 0.0135 & 0.0392 & 0.0257 & $<<0.001$ \\
\hline & Oral region & 0.0028 & 0.0139 & 0.0111 & $<<0.001$ \\
\hline \multirow[t]{4}{*}{ Terrestrial } & Whole skull & 0.0175 & 0.0484 & 0.0309 & $<<0.001$ \\
\hline & Neurocranium & 0.0347 & 0.0628 & 0.0281 & 0.097 \\
\hline & Viscerocranium & 0.0182 & 0.0660 & 0.0478 & $<<0.001$ \\
\hline & Oral region & 0.0060 & 0.0164 & 0.0103 & 0.001 \\
\hline \multirow[t]{4}{*}{ Fossorial } & Whole skull & 0.0198 & 0.0314 & 0.0116 & 0.103 \\
\hline & Neurocranium & 0.0380 & 0.0296 & -0.0089 & 0.454 \\
\hline & Viscerocranium & 0.0180 & 0.0275 & 0.0095 & 0.203 \\
\hline & Oral region & 0.0040 & 0.0143 & 0.0103 & $0.033^{b}$ \\
\hline
\end{tabular}

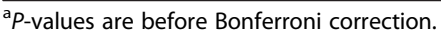

${ }^{\mathrm{b}}$ No longer significant after Bonferroni correction. 


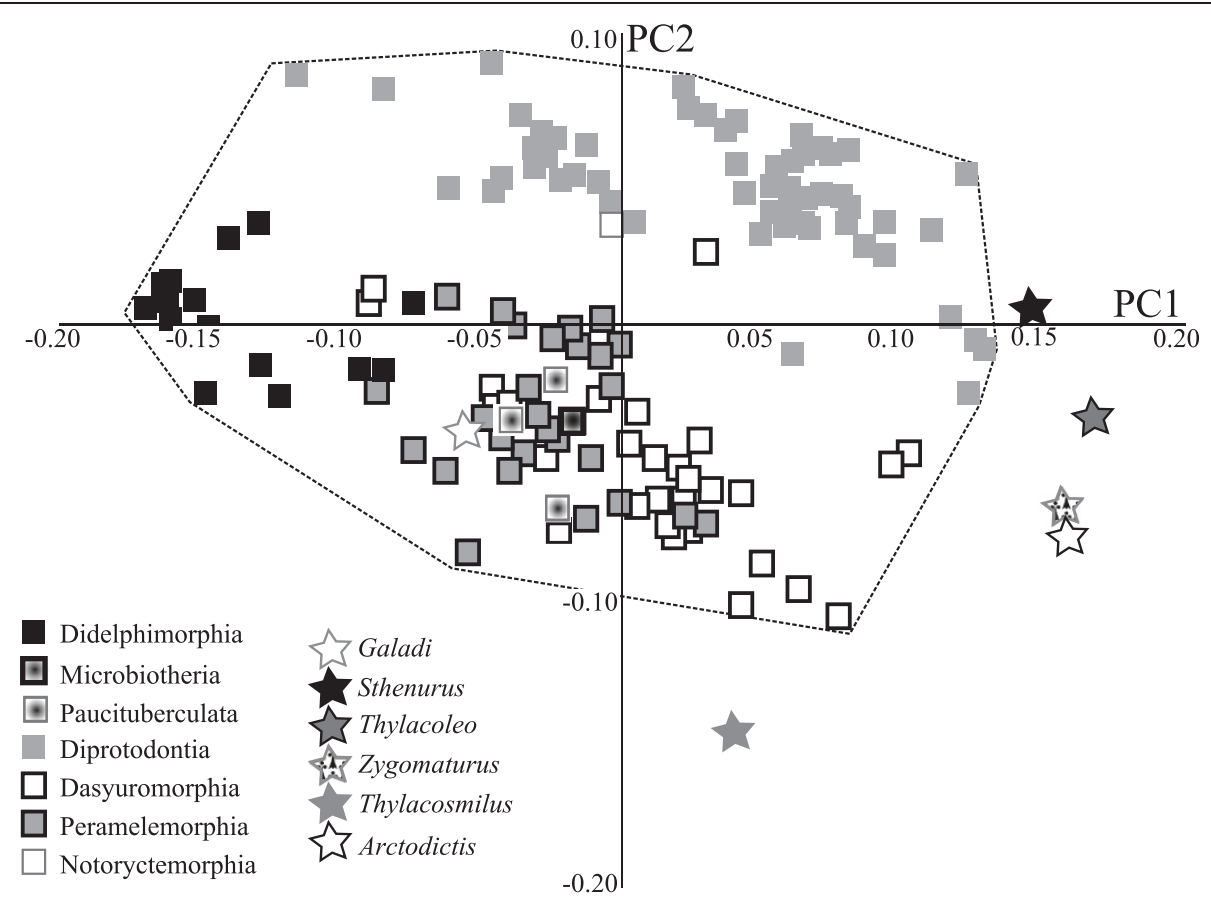

Figure 5 Principal components (PC)1 and PC2 for the extinct and extant metatherians dataset. Squares represent extant taxa, stars represent fossil taxa, and dashed line indicates the range of morphospace occupied by extant metatherians.

functions in isolation from the rest of the quickly developing viscerocranium and the rest of the skull), is related to the evolution of their distinct cranial morphology, relative to that of other marsupials. Future work on peremelemorphian cranial ontogeny thus represents an interesting avenue for research to further address the role of ossification timing and functional constraints on cranial evolution. A specific question of interest is whether peramelemorphians follow or deviate from the developmental trajectory of Monodelphis, discussed above, which is often used to represent a generalized marsupial condition.

It is important to remember that we cannot say for certain whether extinct forms shared the unique developmental strategy of extant marsupials, or indeed, with which group of marsupials they shared most developmental similarities. Of particular interest in this regard are the sparassodonts, included in this analysis and previous studies [21]. These form a group of South American metatherians of uncertain phylogenetic position, although recent analyses have placed them outside of crown marsupials [37]. It is possible that these taxa do not share the same developmental strategy, and thus might not be subject to the same developmental constraints on morphology, as crown marsupials. Nonetheless, the inclusion of fossil taxa did not significantly affect our results, and so we tentatively suggest that the developmental constraint hypothesis may apply to all of the metatherian clades sampled here.

\section{Comparisons with previous studies}

Our findings are consistent with those of Prevosti et al. [22] who also found evidence to support the hypothesis of morphological constraint in the oral region (albeit in the dentary, not tested here) of extant marsupial carnivores. Functionally, this is logical, as the upper and lower jaws should be morphologically coupled for both mechanical and developmental reasons. Conversely, the results of this study concerning the marsupial carnivore/insectivore skull are in contrast to those of Goswami et al. [21], who included a broader sampling of living and extinct insectivorous and carnivorous species. Because our study compared only ecological groupings of recent taxa, it is possible that this disagreement indicates a greater diversity of carnivorous/insectivorous metatherians in the fossil record than in the present. Indeed, many carnivorous marsupials (or, more generally, metatherians) have become extinct relatively recently [38]. The inclusion of six metatherian fossil taxa (three of which are probably

Table 2 Delta variance test results for extant marsupials versus extant marsupials plus fossil metatherian taxa

\begin{tabular}{lllll}
\hline Skull region & Marsupials variance & Marsupials and fossils variance & Delta variance & $\boldsymbol{P}$-value \\
\hline Whole skull & 0.0165 & 0.0187 & 0.0022 & 0.205 \\
\hline
\end{tabular}


carnivorous/insectivorous) with the Recent marsupials in this study similarly did not significantly increase the overall variance. However, ecological groups were not analyzed separately when fossils were included, nor were placentals and metatherians, as no fossil eutherians (the clade including placentals and their stem relatives) were sampled. Thus, the difference in results between these studies may be a reflection of the limited sample sizes of the fossil taxa and the much broader sampling of extant taxa and different ecological groups in the analysis presented here. Nonetheless, developmental constraints may limit variation, but need not represent absolute barriers to evolution. Even if metatherian and placental disparity is comparable in a few ecological groups (possibly carnivorous and fossorial taxa), the results presented here suggest that a developmental constraint has limited marsupial cranial evolution for most, if not all, of the history of this clade.

Expanding these studies to include fossils representing other ecological groups is central to assessing whether fossil metatherians were subject to similar constraints to those found here for extant forms. For example, it has been shown that large placental omnivores, but not hypercarnivores, have been constrained, in terms of taxonomic diversity, on the southern continents since the late Oligocene [39]. Whether such a pattern also applies to metatherians, possibly in combination with the geographic constraint hypothesis discussed further below, is a promising avenue for future study that will benefit from a broadening of focus beyond comparisons of carnivorous fossil metatherians.

\section{The metatherian fossil record and alternative hypotheses for differential mammalian diversity}

It has been suggested that functional requirements around birth bear little relevance to adult metatherian morphology $[13,40]$, and that other factors are primarily responsible for the observed differences in marsupial and placental diversity. The first alternative hypothesis concerns the relative ages of crown placentals and crown marsupials, while an alternative hypothesis is that the diversification of metatherians has been limited by their biogeographical history and resulting 'isolation' on the southern continents.

The earliest metatherians (marsupials and their closest fossil relatives) are known to have existed from the Early Cretaceous of China [4], and may have been restricted to the northern continents, with an especially rich record in North America, until the end Cretaceous, although there are some debated occurrences in Africa and Madagascar $[41,42]$. Non-marsupial metatherians (or possible early didelphimorphs), such as herpetotheriids continued to inhabit the northern continents, although at much reduced numbers, well into the Cenozoic [7,43]. Molecular approaches estimate the first divergences of the extant marsupial clades around $69 \mathrm{Ma}$, with the divergences of the Australian orders occurring around $60 \mathrm{Ma}$ [44]. The first paleontological evidence for the extant orders is found in the Paleocene (around 65 to $63.3 \mathrm{Ma}$ ) of North America with the appearance of the peradectids, the first known members of Didelphimorphia. Didelphimorphia, Paucituberculata, and Microbiotheria appear in the Palaeocene (around 64.5 to $62.5 \mathrm{Ma}$ ) of Brazil. Marsupials first appear in scarce numbers in the fossil record of Australia in the early Eocene [45], but it is not until the prolific Riversleigh deposits of the Oligo-Miocene that all remaining extant marsupial orders (Diprotodontia, Notoryctemorphia, Peramelemorphia, and Dasyuromorphia) appear. However, a more precise understanding of Gondwanan metatherian biogeography, particularly with regard to the biogeographic origin of the enigmatic Microbiotheria (a small clade of South American marsupials that is a sister group to Australodelphia) is yet hindered [46] in large part by an extremely poor pre-Oligocene terrestrial vertebrate record from Australia and Antarctica.

Placental phylogenetics and biogeography are somewhat better understood, with a growing body of evidence over the past decade supporting the division of modern placental clades into four superorders, Afrotheria, Xenarthra, Laurasiatheria, and Euarchontoglires, with the latter two combined in Boreoeutheria [47-53]. This divergence of superorders is thought to have been near-simultaneous, and has been linked, albeit contentiously, to their semiisolation in Africa, South America, and Laurasia, respectively [52] (although this hypothesis is not congruent with the presence of early afrotherians in the fossil record of North America [54]). According to recent molecular divergence date estimates, the placental superorders diverged around 88 to $90 \mathrm{Ma}$, but most extant orders seem to have originated near to or soon after the CretaceousPaleogene extinction, around $65 \mathrm{Ma}[2,55]$. Although there is as yet no confirmed paleontological evidence for crown placentals in the Late Cretaceous (Nishihara et al. [51] Meredith et al. [52], and references therein), crown placentals are known from the earliest Paleocene (around 63 to $64 \mathrm{Ma}$ ) of North America, and are found on most continents by the mid Paleocene.

The difference in timing between the basal divergences of crown marsupials and crown placentals has been suggested as one reason for the lower diversity seen in marsupials [40], although the difference in crown-clade age is out of proportion with the difference in taxonomic diversity between these clades. It has also been suggested that marsupials were hit harder by the K-Pg mass extinction than were placentals (or their respective stem groups), but this has never been explicitly tested. Moreover, paleontological evidence suggests that both groups experienced great losses in diversity, with most Cretaceous metatherian and eutherian families becoming extinct during that event [56-59]. 
The second alternative, the geographic constraint hypothesis, is based on the observation that placentals are currently more taxonomically diverse throughout the northern continents, and that the northern continents have been in more frequent contact during the Cenozoic $[8,10]$. Indeed there are many episodes of dispersal among North America, Asia, and Europe, but both Australia and, until the closure of the Isthmus of Panama (around $3 \mathrm{Ma}$ [59], but see Montes et al. [60]), South America, have been almost entirely isolated since the final breakup of Gondwana and opening of the Drake Passage, around 30 Ma [61]. If competition and faunal exchange drive evolution, then geographic isolation and lack of competition may certainly contribute to the current state of marsupial taxonomic diversity. The relatively low diversities of the 'southern' placental superorders Xenarthra and Afrotheria may provide further evidence for the possible, but as of yet untested, importance of geographic isolation.

More importantly, however, neither clade age nor the geographic constraint hypothesis can account for the differential disparities of the viscerocranium and neurocranium described here. If extrinsic factors are primarily responsible for the low taxonomic diversity and low morphological disparity of marsupials, then all regions of the skull, not just the early-ossifying viscerocranial elements, should show lower disparity in marsupials than in placentals. The results of the study presented here are consistent with the hypothesis of developmental constraint in the marsupial skull, but do not exclude the possibility of some geographical component also limiting metatherian evolution. Ideally, future work combining both aspects would more fully sample from the metatherian fossil record, including that of the northern continents, but at present, there is a paucity of complete and undeformed metatherian cranial material from those regions.

\section{Conclusions}

More fossil data representing the full range of metatherian ecology, as well as quantitative developmental data, are necessary to further test both the hypothesis of cranial constraint in marsupials and the alternative hypothesis. However, the results of this study are consistent with the hypothesis that a developmental constraint imposed by the marsupial reproductive strategy of short gestation and long lactation periods has limited the cranial disparity in this clade of mammals. In particular, the observation that marsupials are less disparate than placentals in viscerocranial morphology, but are equally disparate in neurocranial morphology, is highly suggestive that the early ossification and use of the oral apparatus in marsupials is the specific driver of the differential disparities of these clades. Lastly, our preliminary data for fossil metatherians suggests that this constraint may also have applied to the broader clade and is not limited to crown group marsupials; however, this is a limited sample and should be interpreted with caution.

Future work should endeavor to expand fossil sampling by exploring methods that are not reliant on identifying comparable landmarks across a wide range of taxa, because poor preservation limits the availability of complete specimens and can obscure sutures. Different methods of morphological data capture and analysis are currently being explored in order to enable the inclusion of damaged or partial fossil skulls. These improvements will enable a more robust investigation of this constraint in extinct marsupials and their stem relatives, and will further elucidate the patterns and process that have shaped the evolution of metatherian diversity.

\section{Methods}

\section{Specimens}

Landmark data (Figure 6, Table 3) were collected using a digitizer (Immersion MicroScribe G2X; Immersion Corp., San Jose, CA, USA) and a laser scanner (NextEngine; NextEngine Inc., Santa Monica, CA, USA). Skulls from 125 species of therian mammals (see Additional file 5: Table S5) were used in this study, including where possible a male and a female with the same provenance for each selected species. Species were selected using a randomnumber generator to choose one species from every marsupial genus and one genus from every placental family, in accordance with Wilson and Reeder's mammalian species list [3]. If that species was not available in international museum collections, the next one on the list was selected. Some taxa were excluded from the study because of lack of availability of a complete undamaged adult skull (for example, Tarsipes, the honey-possum), lack of enough clearly homologous landmarks (for example, animals with heavily fused skulls such as bats and some carnivorans, or animals with widely divergent cranial morphology, such as whales), or inability to landmark the skull in just two views. Elephant and rhino skulls were excluded because of their large size, as stitching several 'patches' of overlapping landmarks to fully cover the cranium would have increased the error relative to all other skulls. Although these exclusions primarily involved unusual placentals and thus may have reduced estimates of placental cranial disparity, our sampling did include the vast majority of extant placental diversity, particularly in the terrestrial realm cohabited by marsupials.

\section{Landmarks}

Landmarks were selected to represent clearly homologous points, such as suture junctions or extreme points of curvature, and to fully sample the morphology of the entire skull (Figure 6, Table 3). The number of landmarks used was largely limited by the extent of fusion of the skull bones to one another, limiting the ability to 


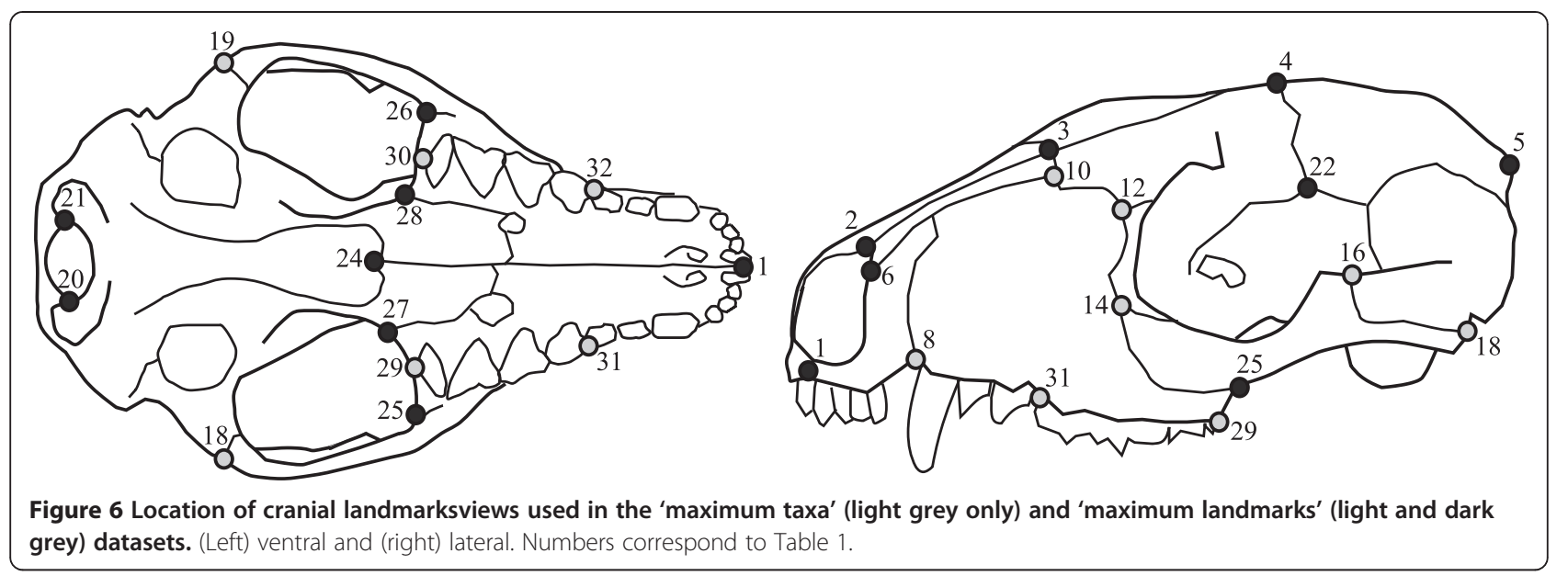

identify sutures. This fusion was mostly a problem in the neurocranium and basicranium, and mainly affected placental musteloid carnivorans and some fossorial forms. Sutures also vary across mammal groups (and sometimes within species or even specimens) in terms of which bones are in contact. The most variable of these sutures were necessarily excluded from this study. Others were included in a subset of the data (see below). In some cases, it was possible to relax the description of the landmark to make it applicable across a larger range of taxa; for example, 'the ventral extent of the frontalparietal suture' does not make it necessary to specify whether this point on the skull is contacting the alisphenoid or squamosal bone.

Three configurations of landmark data were analyzed. A 'maximum landmark' dataset maximized homologous landmarks ( $\mathrm{n}=32$ ) and included 8 (of the 20 extant) placental and all seven marsupial orders, and a total of 104 species. A 'maximum taxa' dataset used a reduced number of landmarks $(\mathrm{n}=16)$ and included 15 (of the

Table 3 Cranial landmark descriptions and assignment to skull regions for disparity analyses ${ }^{\mathrm{a}}$

\begin{tabular}{lll}
\hline Landmark number $^{\mathrm{b}}$ & Landmark description & Skull region \\
\hline $1^{\mathrm{c}, \mathrm{d}}$ & Midline point between the premaxillae and the upper central incisors & Oral \\
\hline $\mathbf{2}^{\mathrm{c} d}$ & Midline anterior-most point of the nasal-nasal suture & Viscerocranium (non-oral) \\
\hline $4^{\mathrm{d}}$ & Midline posterior point of the nasals in contact with frontals & Viscerocranium (non-oral) \\
\hline $5^{\mathrm{c}, \mathrm{d}}$ & Midline posterior point of the frontals in contact with parietals & Neurocranium \\
\hline 6 and $7^{\mathrm{c}, \mathrm{d}}$ & Midpoint at posterior-most extent of vault & Neurocranium \\
\hline 8 and $9^{c}$ & Anterior nasal-premaxilla/maxilla (nasal opening), left and right & Viscerocranium (both oral and non-oral) \\
\hline 10 and 11 & Premaxilla-maxilla suture on the alveolar lateral margin, left and right & Oral \\
\hline 12 and 13 & Nasal-frontal-maxilla/premaxilla suture junction, left and right & Viscerocranium (both oral and non-oral) \\
\hline 14 and 15 & Medial-most maxilla-lacrimal contact, left and right & Viscerocranium (both oral and non-oral) \\
\hline 16 and $17^{c}$ & Lateral most maxilla-lacrimal contact, left and right & Viscerocranium (both oral and non-oral) \\
\hline 18 and $19^{c}$ & Jugal-squamosal (dorsal zygomatic arch), left and right & Viscerocranium (non-oral) \\
\hline 20 and $21^{c, d}$ & Jugal-squamosal (ventral zygomatic arch), left and right & Viscerocranium (non-oral) \\
\hline 22 and $23^{c} c$ & Dorsal most occipital condyle-foramen magnum margin, left and right & Neurocranium \\
\hline $24^{c}$ & Ventral-most extent of frontal-parietal suture, left and right & Neurocranium \\
\hline 25 and $26^{c, d}$ & Midline posterior point of the palatine-palatine suture & Oral \\
\hline 27 and $28^{d}$ & Ventral-most point of the jugal-maxilla suture & Viscerocranium (non-oral) \\
\hline 29 and $30^{c}$ & Posterior-most maxilla-palatine junction on ventral surface, left and right & Oral \\
\hline 31 and $32^{c}$ & Posterior lateral extent of molar row & Oral \\
\hline
\end{tabular}

${ }^{\mathrm{a}}$ Fossil skulls investigated here included the Oligo-Miocene peramelmorphian Galadi speciosus, the Quaternary diprotodontians Sthenurus occidentalis, Zygomaturus trilobus, and Thylacoleo carnifex, and the sparassodonts Thylacosmilus atrox (Miocene) and Arctodictis sinclari (Eocene).

${ }^{\mathrm{b}}$ Numbers correspond to Figure 1.

'Landmarks used in 'maximum fossil' dataset

'Landmarks used 'maximum taxa' dataset. 
20 extant) placental orders and all 7marsupial orders, giving a total of 125 therian species. The 'maximum fossils' dataset used a landmark set $(n=20)$ intermediate between that of the 'maximum landmark' and 'maximum taxa' datasets to optimize the number of fossil taxa that could be compared with Recent marsupial taxa. This last dataset included the six fossil taxa described above (see Additional file 5: Table S5), and 7 extant marsupial orders, to give a total of 82 living and extinct species. All fossils used in this study were considered accurate representations of the original skull shape free from deformation, as they did not exhibit considerable asymmetry. The inclusion of extinct metatherians investigated whether extant diversity is contradicted in the fossil record and is a particular condition of the present. Investigation of extinct eutherian diversity was beyond the scope of this study.

\section{Ecological groups}

The extant 'maximum taxa' landmark data was further separated into eight ecological groups based on dominant diet type, time of activity and habitat, using information sourced from the Animal Diversity Web [62]: folivore, frugivore, carnivore/insectivore, omnivore, nocturnal/crepuscular, arboreal, fossorial, and terrestrial (see Additional file 5: Table S5). Insectivores and carnivores were combined into one dietary group because many insectivorous marsupial taxa also regularly consume small vertebrates and this dietary distinction is largely related to the size of the animal. Because only 11 taxa are known to be crepuscular, and 6 of these are also reported to be nocturnal, the crepuscular and nocturnal taxa were combined into one group. Because only two marsupial taxa (Myrmecobius and Hypsiprymnodon) are truly diurnal, and only two marsupial taxa are undisputedly cathemeral (Aliurops and Dasycercus), these categories were removed from further analysis. Despite exclusion of diurnal and cathemeral taxa, the nocturnal/crepuscular dataset still provided useful comparisons between marsupials and placentals based on time of most frequent activity.

\section{Data analysis}

Prior to all analyses, Procrustes superimposition was used to remove the size and orientation components of the data, leaving only shape. Next, PC analysis was performed to examine distribution and overlap of the clades of interest in the cranial morphospace. Allometry was removed in an attempt to avoid biasing results by the smaller size range and average size of marsupial skulls compared with those of placentals. This was achieved by removing the component of variation explained by difference in centroid size, determined by regressing log centroid size against initial PC scores. The residual of this regression (that is, the components of variation not explained by size alone) were then subjected to a second PC analysis.
Before carrying out comparisons of disparity, sampling issues must be addressed. Variance-based disparity measures, such as those used here, are more robust to sample size than range-based metrics [63], but we further corrected for differences in sample size between the marsupial and placental datasets by bootstrapping male and female marsupials and placentals to the size of the smallest dataset before quantifying and comparing disparity. We conducted 1,000 iterations of the bootstrapping procedure in order to produce results robust to differences in sample size.

To compare cranial disparity between groups across the entire skull and within specific cranial regions, a delta variance test was used. This approach tested for significant differences in the variance of two groups, marsupials and placentals, compared with the variance expected if the taxa sampled were randomly assigned to a group, essentially deciding whether the difference in variance between the marsupial and placental groups is different to that which would be generated by any random grouping of the sampled taxa. To generate the null expectation, the residual Procrustes distances of individual taxa from the mean of each group (after Procrustes superimposition) were randomly permuted and reassigned to the two groups. This process was repeated 1,000 times, and the resulting differences in variance in the permuted datasets were compared with the original differences in variance observed between marsupials and placentals to determine if the observed differences were significantly greater than the random expectation. The following cranial regions were compared: entire cranium, neurocranium, oral region, viscerocranium, and viscerocranium excluding any elements of the oral region. All analyses were conducted using the statistical programming software ' $R$ ' [64] using the software packages shapes [65] and a bind [66], as well as some custom-written code (see supplementary information). Significance was set at $P<0.01$ for all analyses, with a Bonferroni correction of 5 and 36 for the maximum landmarks and maximum taxa (including ecological splits) datasets, respectively, to account for repeated use of the same data.

Phylogenetically corrected analyses were not performed, as the goal of the project was to compare the disparity of two monophyletic sister clades, hence the phylogenetic component of their morphology ws of key interest. Moreover, application of explicitly phylogenetic methods, such as phylogenetic PC analysis, will not change measures of disparity if the full variance in a dataset is considered [67], as was the case in this study.

\section{Additional files}

Additional file 1: Table S1. Principal component (PC) analysis scores for additional PCs. 
Additional file 2: Figure S2. Principal components (PC)1 to PC 4 for taxa grouped by diet as follows: (a,b) frugivores; $(c, d)$ folivores; $(e, f)$ omnivores; and (g,h) carnivores/insectivores. Symbols as in Figure 3.

Additional file 3: Figure S3. Principal components (PC)1 to PC 4 for taxa grouped by habitat as follows: $(\mathrm{a}, \mathrm{b})$ arboreal; $(c, d)$ terrestrial; and $(e, f)$ fossorial. Symbols as in Figure 3.

Additional file 4: Figure S4. Principal components (PC)1 to PC 4 for nocturnal taxa. Symbols as in Figure 3.

Additional file 5: Table S5. List of taxa used in this study and ecological categories used in disparity analyses. *Used in the 'maximum taxa' dataset only.

\section{Competing interests}

The authors declare that they have no competing interests.

\section{Authors' contributions}

CVB designed the analyses, conducted data collection and analyses, and drafted the manuscript; AG conceived of the study, designed the analyses, and drafted the manuscript. All authors read and approved the final manuscript.

\section{Acknowledgements}

We thank MR Sánchez-Villagra, V Weisbecker, PD Polly, S Wroe, N Milne, JA Finarelli, P Upchurch, $\mathrm{H}$ Chatterjee and $\mathrm{K}$ Sears for helpful discussions concerning this work. We also thank the four anonymous reviewer,s whose comments greatly improved this manuscript. Thanks also go to the following people for their invaluable help in accessing collections at the respective museums and institutes: Roberto Portela Miguez and Louise Tomsett at the Natural History Museum, London; Matt Lowe at the Cambridge University Museum of Zoology; Géraldine Veron at the Muséum National d'Histoire Naturelle; Suzanne Peurach at the National Museum of Natural History, Washington DC; Eileen Westwig at the American Museum of Natural History, New York; Bill Simpson at the Field Museum of Natural History, Chicago; Marcelo Reguero at the Museo de La Plata, La Plat; Alejandro Kramarz at the Museo Argentino de Ciencias Naturales Bernardino Rivadavia, Buenos Aires; Dr. Yong Yi Zhen at the Australian Museum, Sydney; Professor Mike Archer and Dr Suzanne Hand at the University of New South Wales, Sydney; MaryAnne Binnie at the South Australia Museum, Adelaide; and Mikael Siversson at the Western Australian Museum, Perth. We gratefully acknowledge support from a Natural Environment Research Council doctoral training grant, an Abbey Research and Collaboration award and a University of London Central Research Fund award.

\section{Author details}

${ }^{1}$ Department of Genetics, Evolution and Environment, University College London, London, UK. ²Department of Earth Sciences, University College London, London, UK.

Received: 25 January 2013 Accepted: 23 April 2013

Published: 26 April 2013

\section{References}

1. Luo Z-X, Yuan C-X, Meng Q-J, Ji Q: A Jurassic eutherian mammal and divergence of marsupials and placentals. Nature 2011, 476:442-445.

2. Dos Reis M, Inoue J, Hasegawa M, Asher RJ, Donoghue PCJ, Reis M, Yang Z: Phylogenomic datasets provide both precision and accuracy in estimating the timescale of placental mammal phylogeny. Proc $R$ Soc Lond B Biol Sci 2012, 279:3491-3500.

3. Wilson DE, Reeder DM: Mammal Species of the World. Baltimore: Johns Hopkins University Press; 2005.

4. Luo Z-X, Ji Q, Wible JR, Yuan C-X: An Early Cretaceous tribosphenic mammal and metatherian evolution. Science 2003, 302:1934-1940.

5. Vullo R, Gheerbrant E, De Muizon C, Néraudeau D: The oldest modern therian mammal from Europe and its bearing on stem marsupial paleobiogeography. Proc Natl Acad Sci USA 2009, 106:19910-19915.

6. Cifelli RL, Davis BM: Paleontology. Marsupial origins. Science 2003, 302:1899-1900.
7. Ladevèze $S$, Smith $R$, Smith $T$ : Reassessment of the morphology and taxonomic status of the earliest herpetotheriid marsupials of Europe. J Mamm Evol 2012, 19:249-261.

8. Lillegraven JA: Biological considerations of the marsupial-placental dichotomy. Evolution 1975, 29:707-722.

9. Lillegraven JA: Biogeographical considerations of the marsupial-placental dichotomy. Annu Rev Ecol Syst 1974, 5:263-283.

10. Sánchez-Villagra MR: Why are there fewer marsupials than placentals? On the relevance of geography and physiology to evolutionary patterns of mammalian diversity and disparity. J Mamm Evol 2012. doi:10.1007/ s10914-012-9220-3

11. Gemmell RT, Veitch C, Nelson J: Birth in marsupials. Comp Biochem Physiol B 2002, 131:621-630.

12. Clark CT, Smith KK: Cranial osteogenesis in Monodelphis domestica (Didelphidae) and Macropus eugenii (Macropodidae). J Morphol 1993, 215:119-149.

13. Kirsch JAW: Biological aspects of the marsupial-placental dichotomy: a reply to Lillegraven. Evolution 1977, 31:898-900.

14. Maier W: Cranial morphology of the therian common ancestor, as suggested by the adaptions of neonate marsupials. In Mammal phylogeny: Mesozoic differentiation, multituberculates, monotremes, early therians, and marsupials. Edited by Szalay FS, Novacek MJ, McKenna MC. New York: Springer; 1993:165-181.

15. Sears KE: Constraints on the morphological evolution of marsupial shoulder girdles. Evolution 2004, 58:2353-2370.

16. Kelly EM, Sears KE: Limb specialization in living marsupial and eutherian mammals: constraints on mammalian limb evolution. J Mammal 2011, 92:1038-1049.

17. Kelly EM, Sears KE: Reduced phenotypic covariation in marsupial limbs and the implications for mammalian evolution. Biol J Linn Soc Lond 2011 102:22-36.

18. Werdelin L: Comparison of skull shape in marsupial and placental carnivores. Aust J Zool 1986, 34:109-117.

19. Werdelin L: Jaw geometry and molar morphology in marsupial carnivores: analysis of a constraint and its macroevolutionary consequences. Paleobiology 1987, 13:342-350.

20. Wroe $\mathrm{S}$, Milne N: Convergence and remarkably consistent constraint in the evolution of carnivore skull shape. Evolution 2007, 61:1251-1260.

21. Goswami A, Milne N, Wroe S: Biting through constraints: cranial morphology, disparity and convergence across living and fossil carnivorous mammals. Proc R Soc Lond B Biol Sci 2011, 278:1831-1839.

22. Prevosti FJ, Turazzini GF, Ercoli MD, Hingst-Zaher E: Mandible shape in marsupial and placental carnivorous mammals: a morphological comparative study using geometric morphometrics. Zool J Linn Soc Lond 2011, 164:836-855.

23. Sánchez-Villagra MR, Goswami A, Weisbecker V, Mock O, Kuratani S: Conserved relative timing of cranial ossification patterns in early mammalian evolution. Evol Dev 2008, 10:519-530.

24. Goswami A: Cranial modularity and sequence heterochrony in mammals. Evol Dev 2007, 9:290-298.

25. Smith KK: Comparative patterns of craniofacial development in eutherian and metatherian mammals. Evolution 1997, 51:1663-1678.

26. Smith KK: Development of craniofacial musculature in Monodelphis domestica (Marsupialia, Didelphidae). J Morphol 1994, 222:149-173.

27. Nunn CL, Smith KK: Statistical analyses of developmental sequences: the craniofacial region in marsupial and placental mammals. Am Nat 1998, 152:82-101.

28. Abdala F, Flores DA, Giannini NP: Postweaning ontogeny of the skull of Didelphis albiventris. J Mammal 2001, 82:190-200.

29. Flores DA, Giannini NP, Abdala F: Cranial ontogeny of Lutreolina crassicaudata (Didelphidae): a comparison with Didelphis albiventris. Acta Theriol (Warsz) 2003, 48:1-9.

30. Flores DA, Giannini N, Abdala F: Comparative postnatal ontogeny of the skull in the australidelphian metatherian Dasyurus albopunctatus (Marsupialia: Dasyuromorpha: Dasyuridae). J Morphol 2006, 267:426-440.

31. Flores DA, Abdala F, Giannini N: Cranial ontogeny of Caluromys philander (Didelphidae: Caluromyinae): a qualitative and quantitative approach. J Mammal 2010, 91:539-550.

32. Giannini N, Abdala F, Flores DA: Comparative postnatal ontogeny of the skull in Dromiciops gliroides (Marsupialia: Microbiotheriidae). Am Mus Novit 2004, 3460:1-17. 
33. Porto A, Oliveira FB, Shirai LT, Conto V, Marroig G: The evolution of modularity in the mammalian skull I: morphological integration patterns and magnitudes. Evol Biol 2009, 36:118-135.

34. Shirai LT, Marroig G: Skull modularity in neotropical marsupials and monkeys: size variation and evolutionary constraint and flexibility. J Exp Zool Part B 2010, 314:663-683.

35. Goswami A, Polly PD, Mock OB, Sánchez-Villagra MR: Shape, variance and integration during craniogenesis: contrasting marsupial and placental mammals. J Evol Biol 2012, 25:862-872.

36. Tyndale-Biscoe H: Life of Marsupials. 2nd edition. Collingwood (Victoria): CSIRO Publishing; 2005.

37. Forasiepi AM: Osteology of Arctodictis sinclairi (Mammalia, Metatheria, Sparassodonta) and phylogeny of Cenozoic metatherian carnivores from South America. Monografias del Museo Argentino de Ciencias Naturales 2009, 6:1-174.

38. Taylor P, Wroe S, Field J, Fullagar R, Lars S: Megafaunal extinction in the late Quaternary and the global overkill hypothesis. Alcheringa 2004, 28:291-331.

39. Van Valkenburgh B: Deja vu: the evolution of feeding morphologies in the Carnivora. Integr Comp Biol 2007, 47:147-163.

40. Simons EL, Bown TM: A new species of peratherium (didelphidae; polyprotodonta): the first African marsupial. J Mammal 1984, 65:539-548.

41. Krause DW: Fossil molar from a Madagascan marsupial. Nature 2001, 412:497-498.

42. Sánchez-Villagra M, Ladevèze $S$, Horovitz I, Argot C, Hooker JJ, Macrini TE, Martin T, Moore-Fay S, De Muizon C, Schmelzle T, Asher RJ: Exceptionally preserved North American Paleogene metatherians: adaptations and discovery of a major gap in the opossum fossil record. Biol Lett 2007, 3:318-322.

43. Nilsson MA, Arnason U, Spencer PBS, Janke A: Marsupial relationships and a timeline for marsupial radiation in South Gondwana. Gene 2004, 340:189-196.

44. Godthelp H, Archer M, Cifelli R: Earliest known Australian Tertiary mammal fauna. Nature 1992, 356:514-516.

45. Beck RMD: An "ameridelphian" marsupial from the early Eocene of Australia supports a complex model of Southern Hemisphere marsupial biogeography. Die Naturwissenschaften 2012, 99:715-729.

46. Madsen O, Scally M, Douady CJ, Kao DJ, DeBry RW, Adkins R, Amrine HM, Stanhope MJ, De Jong WW, Springer MS: Parallel adaptive radiations in two major clades of placental mammals. Nature 2001, 409:610-614.

47. Murphy WJ, Eizirik E, Johnson WE, Zhang YP, Ryder OA, O'Brien SJ: Molecular phylogenetics and the origins of placental mammals. Nature 2001, 409:614-618

48. Murphy WJ, Eizirik E, O'Brien SJ, Madsen O, Scally M, Douady CJ, Teeling E, Ryder OA, Stanhope MJ, De Jong WW, Springer MS: Resolution of the early placental mammal radiation using Bayesian phylogenetics. Science 2001, 294:2348-2351.

49. Springer MS, Stanhope MJ, Madsen O, De Jong WW: Molecules consolidate the placental mammal tree. Trends Ecol Evol 2004, 19:430-438.

50. Kriegs JO, Churakov G, Kiefmann M, Jordan U, Brosius J, Schmitz J: Retroposed elements as archives for the evolutionary history of placental mammals. PLoS Biol 2006, 4:e91.

51. Nishihara H, Maruyama S, Okada N: Retroposon analysis and recent geological data suggest near-simultaneous divergence of the three superorders of mammals. Proc Natl Acad Sci USA 2009, 106:5235-5240.

52. Meredith RW, Janečka JE, Gatesy J, Ryder O, Fisher C, Teeling EC, Goodbla A, Eizirik E, Simão TLL, Stadler T, Rabosky DL, Honeycutt RL, Flynn JJ, Ingram CM, Steiner C, Williams TL, Robinson TJ, Burk-Herrick A, Westerman M, Ayoub N, Springer MS, Murphy WJ: Impacts of the Cretaceous terrestrial revolution and $\mathrm{KPg}$ extinction on mammal diversification. Science 2011, 334:521-524.

53. Zack SP, Penkrot TA, Bloch Jl, Rose KD: Affinities of "hyopsodontids" to elephant shrews and a Holarctic origin of Afrotheria. Nature 2005, 434:497-501.

54. Tavaré S, Marshall CR, Will O, Soligo C, Martin RD: Using the fossil record to estimate the age of the last common ancestor of extant primates. Nature 2002, 416:726-729.

55. Wible JR, Rougier GW, Novacek MJ, Asher RJ: Cretaceous eutherians and Laurasian origin for placental mammals near the $\mathrm{K} / \mathrm{T}$ boundary. Nature 2007, 447:1003-1006.
56. Goswami A, Prasad GVR, Upchurch P, Boyer DM, Seiffert ER, Verma O, Gheerbrant E, Flynn JJ: A radiation of arboreal basal eutherian mammals beginning in the Late Cretaceous of India. Proc Natl Acad Sci USA 2011 108:16333-16338.

57. Goswami A: A dating success story: genomes and fossils converge on placental mammal origins. EvoDevo 2012, 3:18.

58. Rose KD: Introduction. In The Beginning of the Age of Mammals. Baltimore: The Johns Hopkins University Press; 2006:1-21.

59. Keigwin LD: Pliocene closing of the Isthmus of Panama, based on biostratigraphic evidence from nearby Pacific Ocean and Caribbean Sea cores. Geology 1978, 6:630-634.

60. Montes C, Cardona A, McFadden R, Moron SE, Silva C, Restrepo-Moreno S, Ramirez D, Hoyos N, Wilson J, Farris D, Bayona G, Jaramillo C, Valencia V, Bryan J, Flores J: Evidence for middle Eocene and younger land emergence in central Panama: implications for Isthmus closure. Geol Soc Am Bull 2012, 124:780-799.

61. Barker PF, Burrell J: The problem of drake passage opening has significance outside the field of regional tectonics: the creation of a continuous circumAntarctic deep water path in the zone of the mid-latitude westerly winds by separation from Antarctica of south America. Mar Geo/ 1977, 25:15-34.

62. The Animal Diversity Web. http://animaldiversity.org.

63. Foote M: The evolution of morphological diversity. Ann Rev Ecol Syst 1997, 28:129-152

64. R Core Team R: A Language and Environment for Statistical Computing. $R$ Foundation for Statistical Computing 2012, http://www.R-project.org/.

65. Dryden I: Shapes: Statistical shape analysis. 2009, http://CRAN.R-project. org/package=shapes.

66. Plate T, Heiberger R: Abind: Combine multi-dimensional arrays. 2011 http://CRAN.R-project.org/package=abind.

67. Polly PD, Lawing AM, Fabre A-C, Goswami A: Phylogenetic principal components analysis and geometric morphometrics. Hystrix 2002, 11:9-25.

doi:10.1186/1741-7007-11-52

Cite this article as: Bennett and Goswami: Statistical support for the hypothesis of developmental constraint in marsupial skull evolution. BMC Biology 2013 11:52.

\section{Submit your next manuscript to BioMed Central and take full advantage of:}

- Convenient online submission

- Thorough peer review

- No space constraints or color figure charges

- Immediate publication on acceptance

- Inclusion in PubMed, CAS, Scopus and Google Scholar

- Research which is freely available for redistribution 